\title{
Current medulloblastoma subgroup specific clinical trials
}

\author{
Eric M. Thompson ${ }^{1,2,3,4}$, David Ashley ${ }^{1,2,3}$, Daniel Landi ${ }^{1,2,3}$ \\ ${ }^{1}$ Department of Neurosurgery, ${ }^{2}$ Preston Robert Tisch Brain Tumor Center, ${ }^{3}$ Department of Pediatrics, ${ }^{4}$ Department of Pathology, Duke University, \\ Durham, NC, USA \\ Contributions: (I) Conception and design: EM Thompson; (II) Administrative support: None; (III) Provision of study materials or patients: None; (IV) \\ Collection and assembly of data: EM Thompson; (V) Data analysis and interpretation: All authors; (VI) Manuscript writing: All authors; (VII) Final \\ approval of manuscript: All authors. \\ Correspondence to: Eric M. Thompson, MD. Duke University Medical Center, Box 3272, Durham, NC 27710, USA. Email: eric.thompson@duke.edu.
}

\begin{abstract}
Medulloblastoma is a heterogeneous disease with at least four distinct molecular subgroups: wingless (WNT), sonic hedgehog (SHH), Group 3, and Group 4. Recently there has been considerable progress defining the molecular drivers and prognostic factors of each subgroup. However, this information has only rarely been used to stratify risk or impact treatment. The purpose of this work is to provide an update on current clinical trials that provide molecularly stratified treatment paradigms. A search was conducted on ClinicalTrials.gov using the following search terms: "medulloblastoma and subgroup", "medulloblastoma and SHH", "medulloblastoma and WNT", and "medulloblastoma and Non-WNT/ Non-SHH". This search resulted in nine distinct clinical trials, five for newly diagnosed medulloblastoma and four for recurrent medulloblastoma. Four trials for newly diagnosed medulloblastoma had a component of craniospinal irradiation reduction for patients with WNT medulloblastoma. Molecularly stratified trials for recurrent medulloblastoma largely focus on SHH. As these trials are ongoing, there are limited data available. A trial in which newly-diagnosed WNT patients received modest chemotherapy without radiation has been closed to accrual due to several early failures. Phase II trials evaluating vismodegib for $\mathrm{SHH}$ medulloblastoma in children and adults have been disappointing. In conclusion, although there is an expanding array of clinical trials which incorporate molecular data in prescribing treatment for newlydiagnosed and recurrent medulloblastoma, treatments for these diseases are fairly uniform, with craniospinal radiation dose being the main variable. As the drivers of the distinct subgroups and their associated prognoses are better elucidated, future clinical trials and novel targeted agents are needed to improve outcomes and reduce toxicity where feasible.
\end{abstract}

Keywords: Medulloblastoma; wingless (WNT); sonic hedgehog (SHH); Group 3; Group 4; clinical trial

Submitted Oct 30, 2019. Accepted for publication Feb 25, 2020.

doi: $10.21037 /$ tp.2020.03.03

View this article at: http://dx.doi.org/10.21037/tp.2020.03.03

\section{Introduction}

Medulloblastoma is the most common malignant pediatric brain tumor, with approximately 1,800 new cases diagnosed in the United States every year (1). Traditionally, treatment of medulloblastoma consists of maximum safe surgical resection followed by craniospinal irradiation (CSI) and chemotherapy. Residual disease after surgery $>1.5 \mathrm{~cm}^{2}$, anaplastic histology, metastatic disease or positive cytology, and MYC amplification define high-risk disease, which is treated with 36 Gy CSI + boosts to the posterior fossa and bulk disease. Overall, with adjuvant radiation and chemotherapy, patients have a 5 -year overall survival (OS) of $60 \%$ to $85 \%$ (2), however, nearly all patients experience therapy-related toxicity. The St Jude medulloblastoma protocols prescribe 23.4 Gy CSI plus minimum 54 Gy posterior fossa boost for average risk patients, which is increased to 36Gy CSI for high risk disease. Adjuvant chemotherapy includes $12-16 \mathrm{~g} / \mathrm{m}^{2}$ cyclophosphamide and $300 \mathrm{mg} / \mathrm{kg}$ cisplatin over 4 cycles. Grade 3 or 4 ototoxicity 
occurs in over $18 \%$ of patients (3), endocrinopathies in over $50 \%$ of patients (4), and permanent cognitive decline in the majority of patients, particularly younger children (5). Moreover, up to $10 \%$ develop secondary malignancies as adults (6). Therefore, reducing the effects of toxic adjuvant therapies without negatively impacting efficacy is paramount.

Medulloblastoma is not a single entity, but rather is comprised of at least four molecular subgroups (7) and 12 subtypes (8). These four molecular subgroups which include wingless (WNT), sonic hedgehog (SHH), Group 3 , and Group 4 and have distinct features including tumor drivers, prognosis, and patient demographics (7). Given these differences in subgroups and the high incidence of adjuvant therapy-related morbidity, there has recently been significant interest in tailoring adjuvant therapy with the goal of reducing toxic chemotherapy and radiation while optimizing patient outcomes. The purpose of this paper is to provide an update on current clinical medulloblastoma trials that stratify adjuvant treatment based upon molecular subgroup.

\section{Methods}

A search was conducted on ClinicalTrials.gov on 8/21/19 using the following search terms: "medulloblastoma and subgroup", "medulloblastoma and SHH", "medulloblastoma and WNT", and "medulloblastoma and Non-WNT/ Non-SHH". These resulted in 13, 9, 5, and 2 clinical trials respectively with overlap amongst the results. Previously completed studies and studies not captured by the above search results were not included.

\section{Results}

A brief summary of the results can be found in Table 1 .

\section{Trials for newly diagnosed medulloblastoma}

Children with WNT medulloblastoma have by far the most favorable outcomes of all medulloblastoma subgroups (9). Subsequently, there is considerable interest in de-escalating treatment these patients receive in the adjuvant setting in order to reduce therapy-related morbidity. One of the largest studies currently open is, "A Clinical and Molecular Risk-Directed Therapy for Newly Diagnosed Medulloblastoma," otherwise known as SJMB 12 (10). The study plans to enroll 625 participants based on their subgroup, WNT (Strata W), SHH (Strata S), and non-
WNT/non-SHH (Strata N). Stratum W1 (low risk) will undergo reduced CSI dose (15/51 Gy), Stratum W2 (atypical) will undergo standard CSI (23.4/54 Gy), and Stratum W3 (high risk) will undergo high CSI (36-39.6/54 Gy). Strata $\mathrm{S} 1, \mathrm{~N} 1$, and N2 will receive 23.4/54 Gy while S2 and N3 will receive 36-39.6/54 Gy. In addition to cyclophosphamide, cisplatin, and vincristine, patients in SHH stratum with skeletal maturity receive the hedgehog pathway inhibitor, vismodegib, as this agent causes premature growth plate fusion in pre-pubertal children. High-risk patients in the non-WNT/non-SHH stratum receive the synthetic pyrimidine nucleoside prodrug, gemcitabine, and the folate antimetabolite, pemetrexed, but these are conventional chemotherapies without specific subgroup rationale. In addition to the typical outcome measures, patients will undergo a battery of cognitive and quality of life assessments.

The Children's Oncology Group (COG) trial, "A Phase 2 Study of Reduced Therapy for Newly Diagnosed Average-Risk WNT-Driven Medulloblastoma Patients," enrolls only WNT medulloblastoma patients and prescribes 18 Gy CSI and a smaller tumor bed irradiation of $36 \mathrm{~Gy}$ in lieu of a boost to the entire posterior fossa, as well as reduced cisplatin, vincristine, and lomustine doses (11). A study coordinated by the Sidney Kimmel Comprehensive Cancer Center at Johns Hopkins, "Pilot Study Assessing the Feasibility of a Surgery and Chemotherapy-Only Approach in the Upfront Therapy of Children With Wnt Positive Standard Risk Medulloblastoma," enrolled only low risk WNT patients with $<1.5 \mathrm{~cm}^{2}$ residual disease and no metastases (12). Patient received no CSI and received a modest chemotherapy regimen including lomustine, vincristine, and cisplatin followed by cyclophosphamide, vincristine, and mesna, a bladder protective agent. Unfortunately, enrollment is currently suspended due to two patients experiencing both local and disseminated recurrence at 3 and 6 months (13). Preliminarily, it appears that modest-dose chemotherapy without radiation is inadequate to treat average risk WNT medulloblastoma.

The International Society of Paediatric Oncology (SIOP)'s study, "An International Prospective Study on Clinically Standard-risk Medulloblastoma in Children Older Than 3 to 5 Years With Low-risk Biological Profile (PNET 5 MB-LR) or Average-risk Biological Profile (PNET 5 MB-SR)," stratifies patients into low risk and standard risk arms (14). The low risk stratum is comprised of WNT patients that receive 18 Gy of CSI and reduced doses of cisplatin, lomustine, and vincristine followed by 
Table 1 Clinical trial summary

\begin{tabular}{|c|c|c|c|c|}
\hline Trial & $\begin{array}{l}\text { ClinicalTrials.gov } \\
\text { Number }\end{array}$ & $\begin{array}{c}\text { Target } \\
\text { enrollment }\end{array}$ & Key features & Available results \\
\hline \multicolumn{5}{|l|}{ Newly diagnosed } \\
\hline \multirow{2}{*}{$\begin{array}{l}\text { A Clinical and Molecular Risk-Directed Therapy for } \\
\text { Newly Diagnosed Medulloblastoma (SJMB 12) }\end{array}$} & \multirow{2}{*}{ NCT01878617 } & \multirow{2}{*}{625} & Vismodegib for SHH patients & \\
\hline & & & $\begin{array}{l}\text { Pemetrexed and Gemcitabine for } \\
\text { non-WNT/non-SHH patients }\end{array}$ & \\
\hline \multirow{3}{*}{$\begin{array}{l}\text { A Phase } 2 \text { Study of Reduced Therapy for Newly } \\
\text { Diagnosed Average-Risk WNT-Driven } \\
\text { Medulloblastoma Patients (COG ACNS1422) }\end{array}$} & \multirow{3}{*}{ NCT02724579 } & \multirow{3}{*}{45} & Reduced CSI & \\
\hline & & & $\begin{array}{l}\text { Limited targeted radiation boost to } \\
\text { the tumor bed }\end{array}$ & \\
\hline & & & $\begin{array}{l}\text { Reduced cisplatin, vincristine, and } \\
\text { lomustine }\end{array}$ & \\
\hline $\begin{array}{l}\text { An International Prospective Study on Clinically } \\
\text { Standard-risk Medulloblastoma in Children Older Than } \\
3 \text { to } 5 \text { Years With Low-risk Biological Profile (PNET } 5 \\
\text { MB-LR) or Average-risk Biological Profile (PNET } 5 \\
\text { MB-SR) }\end{array}$ & NCT02066220 & 360 & $\begin{array}{l}\text { Reduced CSI and reduced } \\
\text { cisplatin, vincristine, lomustine } \\
\text { followed by cyclophosphamide and } \\
\text { vincristine for WNT patients }\end{array}$ & \\
\hline \multirow[b]{2}{*}{$\begin{array}{l}\text { HeadStart4: Newly Diagnosed Children }(<10 \text { y/o) With } \\
\text { Medulloblastoma and Other CNS Embryonal Tumors } \\
\text { Clinical and Molecular Risk-Tailored Intensive and } \\
\text { Compressed Induction Chemotherapy Followed by } \\
\text { Consolidation With Randomization to Either Single } \\
\text { Cycle or to Three Tandem Cycles of Marrow-Ablative } \\
\text { Chemotherapy With Autologous Hematopoietic } \\
\text { Progenitor Cell Rescue }\end{array}$} & \multirow[t]{2}{*}{ NCT02875314 } & \multirow[t]{2}{*}{250} & \multirow[b]{2}{*}{$\begin{array}{l}\text { Only open to patient } \leq 10 \text { years old } \\
\text { After induction chemotherapy, } \\
\text { non-WNT/non-SHH randomized to } \\
\text { possible receive increased } \\
\text { chemotherapy compared to WNT/ } \\
\text { SHH patients }\end{array}$} & \\
\hline & & & & \\
\hline \multirow{2}{*}{$\begin{array}{l}\text { St. Jude ELIOT: Phase } 1 \text { Evaluation of LY2606368, } \\
\text { a Molecularly-Targeted CHK1/2 Inhibitor Therapy, in } \\
\text { Combination With Cyclophosphamide or } \\
\text { Gemcitabine for Children and Adolescents With } \\
\text { Refractory or Recurrent Group 3/Group } 4 \text { or SHH } \\
\text { Medulloblastoma Brain Tumors }\end{array}$} & \multirow[t]{2}{*}{ NCT04023669 } & \multirow[t]{2}{*}{100} & \multicolumn{2}{|l|}{$\begin{array}{l}\text { Not open to patients with recurrent } \\
\text { WNT medulloblastoma }\end{array}$} \\
\hline & & & \multicolumn{2}{|l|}{$\begin{array}{l}\text { Two treatment groups: Group3/4/ } \\
\text { SHH (prexasertib and } \\
\text { cyclophosphamide) and Group 3/4 } \\
\text { (prexasertib and gemcitabine) }\end{array}$} \\
\hline $\begin{array}{l}\text { PBTC-053: A Pediatric Brain Tumor Consortium } \\
\text { Phase I/II and Surgical Study of CX-4945 in Patients } \\
\text { With Recurrent SHH Medulloblastoma }\end{array}$ & NCT03904862 & 60 & $\begin{array}{l}\text { Only open to patients with } \mathrm{SHH} \\
\text { medulloblastoma }\end{array}$ & \\
\hline $\begin{array}{l}\text { An International, Randomized, Open-label Phase I/II } \\
\text { Study of Vismodegib in Combination With } \\
\text { Temozolomide Versus Temozolomide Alone in Adult } \\
\text { Patients With Recurrent or Refractory } \\
\text { Medulloblastomas Presenting an Activation of the } \\
\text { Sonic Hedgehog (SHH) Pathway (MEVITEM) }\end{array}$ & NCT01601184 & 24 & $\begin{array}{l}\text { Open to SHH adult patients only } \\
\text { Not currently enrolling patients }\end{array}$ & $\begin{array}{l}\text { Terminated due } \\
\text { to lack of success } \\
\text { at first stage of } \\
\text { Phase II }\end{array}$ \\
\hline
\end{tabular}


cyclophosphamide and vincristine whereas standard risk patients receive 23.4 Gy CSI.

Nationwide Children's Hospital is sponsoring Headstart IV: "HeadStart4: Newly Diagnosed Children ( $<10$ y/o) With Medulloblastoma and Other CNS Embryonal Tumors Clinical and Molecular Risk-Tailored Intensive and Compressed Induction Chemotherapy Followed by Consolidation With Randomization to Either Single Cycle or to Three Tandem Cycles of Marrow-Ablative Chemotherapy With Autologous Hematopoietic Progenitor Cell Rescue (15)." Patients with non-WNT/non-SHH medulloblastoma receive three (if remission is achieved) to five cycles of vincristine, cisplatin, cyclophosphamide, etoposide, high-dose methotrexate, then are randomized into either a single cycle of carboplatin, thiotepa, and etoposide or tandem three cycles of carboplatin and thiotepa. Patients with WNT or SHH medulloblastoma receive the same three to five cycles induction chemotherapy as the non-WNT/non-SHH but do not undergo randomization and receive a single consolidation cycle of carboplatin, thiotepa, and etoposide with autologous stem cell infusion.

\section{Trials for recurrent medulloblastoma}

St. Jude Children's Research Hospital is conducting a clinical trial for patients with recurrent medulloblastoma, "Molecularly-Driven Doublet Therapy for All Children With Refractory or Recurrent CNS Malignant Neoplasms and Young Adults With Refractory or Recurrent SHH Medulloblastoma." Stratum A includes patients with recurrent/refractory Group 3 and Group 4 medulloblastoma (16). These patients are treated with ribociclib, a cyclin D1/CDK4/6 inhibitor, and gemcitabine. Stratum B includes patients with recurrent/refractory SHH or WNT medulloblastoma; these patients receive ribociclib and the MEK1/2 inhibitor, trametinib. Stratum $\mathrm{C}$ includes patients with recurrent/refractory $\mathrm{SHH}$ medulloblastoma. In contrast to Stratum B, Stratum C patients must be off any smoothened inhibitor for more than 6 months, must have tumor with $9 \mathrm{q}$ loss or PTCH mutation, and be skeletally mature. These patients receive ribociclib and, the smoothened antagonist, sonidegib. Notably, Stratum A will also enroll patients with recurrent ependymoma while Stratum B will also enroll patients with recurrent malignant glioma. In addition to the aforementioned study, St. Jude is also conducting another trial for recurrent medulloblastoma, "St. Jude ELIOT:
Phase 1 Evaluation of LY2606368, a MolecularlyTargeted CHK1/2 Inhibitor Therapy, in Combination With Cyclophosphamide or Gemcitabine for Children and Adolescents With Refractory or Recurrent Group 3/ Group 4 or SHH Medulloblastoma Brain Tumors (17).” This study includes two strata. Stratum A includes patients with recurrent/refractory Group 3, Group 4, SHH, and indeterminate subgroup medulloblastoma. These patients receive the cell cycle checkpoint kinase $1 / 2$ inhibitor, prexasertib, and cyclophosphamide. Stratum B will include patients with recurrent/refractory Group 3 and Group 4 medulloblastoma. These patients receive prexasertib and gemcitabine. Notably, this trial is not open to patients with recurrent WNT medulloblastoma.

Two clinical trials specifically target recurrent $\mathrm{SHH}$ medulloblastoma. The Pediatric Brain Tumor Consortium's trial, "PBTC-053: A Pediatric Brain Tumor Consortium Phase I/ II and Surgical Study of CX-4945 in Patients With Recurrent SHH Medulloblastoma (18)," enrolls patients with recurrent $\mathrm{SHH}$ into one of three arms. All patients receive oral silmitasertib sodium, a casein kinase (CK) 2 inhibitor. Phase I enrolls skeletally immature patients (age 3 and above), Phase II enrolls skeletally mature patients, and the surgical arm enrolls patients from Phase I or II who are candidates for surgery. Another study for patients with recurrent $\mathrm{SHH}$ medulloblastoma is titled, "An International, Randomized, Open-label Phase I/II Study of Vismodegib in Combination With Temozolomide Versus Temozolomide Alone in Adult Patients With Recurrent or Refractory Medulloblastomas Presenting an Activation of the Sonic Hedgehog (SHH) Pathway $(19,20)$." This nowterminated study treated patients with vismodegib plus temozolomide versus temozolomide alone. The final results of this trial have not been published. However, the authors had stated that it "would be uninteresting if 6-month nonprogression rate $\leq 30 \%$ and promising if $\geq 55 \%$ and using Simon's optimal two-stage design (type I error rate: $5 \%$, power: $80 \%$ ) (20)." The trial was terminated because, "The number of successes is not reached at the end of first stage of the phase II (19)".

\section{Discussion}

In this review, we have highlighted nine clinical trials which use medulloblastoma molecular subgrouping to tailor adjuvant therapies. It should be noted that this is not intended to be a comprehensive list of all such trials, just those currently open and captured by the search terms 
noted in Methods. For an excellent review of the scientific rationale behind targeted therapies, please refer to Liu et al. (21). Further, Keiran published an excellent review of studies specifically targeting the SHH pathway (22).

Future studies will undoubtedly offer more targeted therapies as preclinic work continues to drive the field forward. Recent insights into the clinical importance of surgical tumor extent of resection $(9,23)$ have the potential to alter what is considered high-risk disease, thereby further reducing the amount of CSI and adjuvant chemotherapy in patients with minimal residual disease. As the pathogenesis and tumor-drivers of Group 3 and Group 4 continue to be better defined $(21,24-26)$, future trials of targeted agents may contribute to improved survival and quality of life. As the aforementioned trials mature, these results may impact and inform future iterations of moleculary stratified trials, hopefully including less toxic, targeted therapies for medulloblastoma.

\section{Acknowledgments}

Funding: None.

\section{Footnote}

Conflicts of Interest: All authors have completed the ICMJE uniform disclosure form (available at http://dx.doi. org/10.21037/tp.2020.03.03). EMT serves as the unpaid editorial board member of Translational Pediatrics from Jul 2019 to Jun 2021. DA reports personal fees from ISTARI ONCOLOGY, INC., personal fees from JACKSON LABORATORY, during the conduct of the study; other from DIVERSE BIOTECH, outside the submitted work. The other author has no conflicts of interest to declare.

Ethical Statement: The authors are accountable for all aspects of the work in ensuring that questions related to the accuracy or integrity of any part of the work are appropriately investigated and resolved.

Open Access Statement: This is an Open Access article distributed in accordance with the Creative Commons Attribution-NonCommercial-NoDerivs 4.0 International License (CC BY-NC-ND 4.0), which permits the noncommercial replication and distribution of the article with the strict proviso that no changes or edits are made and the original work is properly cited (including links to both the formal publication through the relevant DOI and the license). See: https://creativecommons.org/licenses/by-nc-nd/4.0/.

\section{References}

1. Ostrom QT, Gittleman H, Truitt G, et al. CBTRUS Statistical Report: Primary Brain and Other Central Nervous System Tumors Diagnosed in the United States in 2011-2015. Neuro Oncol 2018;20:iv1-iv86.

2. Martin AM, Raabe E, Eberhart C, et al. Management of pediatric and adult patients with medulloblastoma. Curr Treat Options Oncol 2014;15:581-94.

3. Paulino AC, Lobo M, Teh BS, et al. Ototoxicity after intensity-modulated radiation therapy and cisplatin-based chemotherapy in children with medulloblastoma. Int J Radiat Oncol Biol Phys 2010;78:1445-50.

4. Yock TI, Yeap BY, Ebb DH, et al. Long-term toxic effects of proton radiotherapy for paediatric medulloblastoma: a phase 2 single-arm study. Lancet Oncol 2016;17:287-98.

5. Mulhern RK, Palmer SL, Merchant TE, et al. Neurocognitive consequences of risk-adapted therapy for childhood medulloblastoma. J Clin Oncol 2005;23:5511-9.

6. Christopherson KM, Rotondo RL, Bradley JA, et al. Late toxicity following craniospinal radiation for early-stage medulloblastoma. Acta Oncol 2014;53:471-80.

7. Taylor MD, Northcott PA, Korshunov A, et al. Molecular subgroups of medulloblastoma: the current consensus. Acta Neuropathol 2012;123:465-72.

8. Cavalli FMG, Remke M, Rampasek L, et al. Intertumoral Heterogeneity within Medulloblastoma Subgroups. Cancer Cell 2017;31:737-54.e6.

9. Thompson EM, Hielscher T, Bouffet E, et al. Prognostic value of medulloblastoma extent of resection after accounting for molecular subgroup: a retrospective integrated clinical and molecular analysis. Lancet Oncol 2016;17:484-95.

10. ClinicalTrials.gov. A Clinical and Molecular Risk-Directed Therapy for Newly Diagnosed Medulloblastoma. 2019. Available online: https://clinicaltrials.gov/ct2/show/ NCT01878617. Accessed 9/13/19.

11. ClinicalTrials.gov. Reduced Craniospinal Radiation Therapy and Chemotherapy in Treating Younger Patients With Newly Diagnosed WNT-Driven Medulloblastoma. 2019. Available online: https://www.clinicaltrials.gov/ct2/ show/NCT02724579?term=NCT02724579\&rank=1. Accessed 9/13/19.

12. ClinicalTrials.gov. Study Assessing the Feasibility of a 
Surgery and Chemotherapy-Only in Children With Wnt

Positive Medulloblastoma. 2019. Available online: https:// clinicaltrials.gov/ct2/show/NCT02212574. Accessed 9/13/19.

13. Cohen K, Bandopadhayay P, Chi S, et al. MEDU-34. Pilot study of a surgery and chemotherapy-only approach in the upfront therapy of children with Wnt-positive standard risk medulloblastoma. Neuro Oncol 2019;21:ii110.

14. ClinicalTrials.gov. International Society of Paediatric Oncology (SIOP) PNET 5 Medulloblastoma. 2019. Available online: https://clinicaltrials.gov/ct2/show/ NCT02066220. Accessed 9/13/19.

15. ClinicalTrials.gov. HeadStart4: Newly Diagnosed Children (<10 y/o) With Medulloblastoma and Other CNS Embryonal Tumors. 2019. Available online: https:// clinicaltrials.gov/ct2/show/NCT02875314. Accessed 9/16/19.

16. ClinicalTrials.gov. SJDAWN: St. Jude Children's Research Hospital Phase 1 Study Evaluating Molecularly-Driven Doublet Therapies for Children and Young Adults With Recurrent Brain Tumors. 2019. Available online: https:// clinicaltrials.gov/ct2/show/NCT03434262.

17. ClinicalTrials.gov. Evaluation of LY2606368 Therapy in Combination With Cyclophosphamide or Gemcitabine for Children and Adolescents With Refractory or Recurrent Group 3/Group 4 or SHH Medulloblastoma Brain Tumors. 2019. Available online: https://clinicaltrials.gov/ ct2/show/NCT04023669. Accessed 9/16/19.

18. ClinicalTrials.gov. Testing the Safety and Tolerability of CX-4945 in Patients With Recurrent Medulloblastoma Who May or May Not Have Surgery. 2019. Available online: https://clinicaltrials.gov/ct2/show/NCT03904862.
Accessed 9/16/19.

19. ClinicalTrials.gov. Study of Vismodegib in Combination With Temozolomide Versus Temozolomide Alone in Patients With Medulloblastomas With an Activation of the Sonic Hedgehog Pathway. 2019. Available online: https:// clinicaltrials.gov/ct2/show/NCT01601184. Accessed 9/16/19.

20. Frappaz D, Chinot OL, Meyronet D, et al. MEVITEM: A European, randomized, open-label Phase I/II of vismodegib in combination with temozolomide versus temozolomide alone in adult patients with recurrent/ refractory medulloblastoma presenting an activation of the Sonic Hedgehog pathway. J Clin Oncol 2016;34:TPS2083.

21. Liu KW, Pajtler KW, Worst BC, et al. Molecular mechanisms and therapeutic targets in pediatric brain tumors. Sci Signal 2017;10. doi: 10.1126/scisignal.aaf7593.

22. Kieran MW. Targeted treatment for sonic hedgehogdependent medulloblastoma. Neuro Oncol 2014;16:1037-47.

23. Thompson EM, Bramall A, Herndon JE, 2nd, et al. The clinical importance of medulloblastoma extent of resection: a systematic review. J Neurooncol 2018;139:523-39.

24. Thompson EM, Keir ST, Venkatraman T, et al. The role of angiogenesis in Group 3 medulloblastoma pathogenesis and survival. Neuro Oncol 2017;19:1217-27.

25. Vladoiu MC, El-Hamamy I, Donovan LK, et al. Childhood cerebellar tumours mirror conserved fetal transcriptional programs. Nature 2019;572:67-73.

26. Hovestadt V, Smith KS, Bihannic L, et al. Resolving medulloblastoma cellular architecture by single-cell genomics. Nature 2019;572:74-9.
Cite this article as: Thompson EM, Ashley D, Landi D. Current medulloblastoma subgroup specific clinical trials. Transl Pediatr 2020;9(2):157-162. doi: 10.21037/tp.2020.03.03 